\title{
Phrenic Nerve Pacing: Current Concepts
}

\author{
Jorge F. Velazco, Shekhar Ghamande and Salim Surani
}

Additional information is available at the end of the chapter

http://dx.doi.org/10.5772/48808

\section{Introduction}

Anatomy: The diaphragm is the main inspiratory muscle in mammals, it is a highly active muscle with a duty cycle of $30-40 \%$, and therefore, the diaphragm may be particularly susceptible to inactivity or disuse (1). It is innervated by the phrenic nerves that arise from the nerve roots $\mathrm{C} 3$ through $\mathrm{C} 5$, and is primarily composed of fatigue-resistant slow-twitch type I and fast-twitch IIa myofibers. As the diaphragm contracts, the abdominal contents are displaced caudally, abdominal pressure increases and the lower ribcage expands (2). The phrenic nerve contains sensory, motor and sympathetic nerve fibers, and it supplies motor supply to the diaphragm and sensory supply to the central tendon (3). Both phrenic nerves run along the anterior scalene muscle deep to the carotid sheath. The right phrenic nerve passes over the brachiocephalic artery and posterior to the subclavian vein and over the right atrium. It enters the diaphragm at the level of T8 vertebrae. The left phrenic nerve also runs posterior to the left subclavian vein and passes over the pericardium of the left ventricle and penetrates the left hemidiaphragm separately (3). See Figures 1 and 2.

Pathophysiology: Diaphragmatic dysfunction can manifest as weakness or paralysis, and is related to different entities. Patients with bilateral diaphragmatic paralysis are more likely to have symptoms like dyspnea or recurrent respiratory failure; other complications include subsegmental atelectasis and infections of lower respiratory tract (2). See Figure 3.

Of the 11,000 new cases of spinal cord injury (SCI), approximately one half $(56.4 \%)$ are sustained at the cervical level (4). Currently, the average age of injury is 37.6 , and about $80 \%$ are male (5). Cervical SCI often leads to an interruption of the descending bulbospinal respiratory pathways, resulting in respiratory muscle paresis and/or paralysis. The more rostral the level of injury the greater the likelihood of a major respiratory compromise, however the most caudal segment of the spinal cord with normal motor function defines the motor level of injury. The injuries above the level of the phrenic motoneurons $(\mathrm{C} 3, \mathrm{C} 4, \mathrm{C} 5)$ 


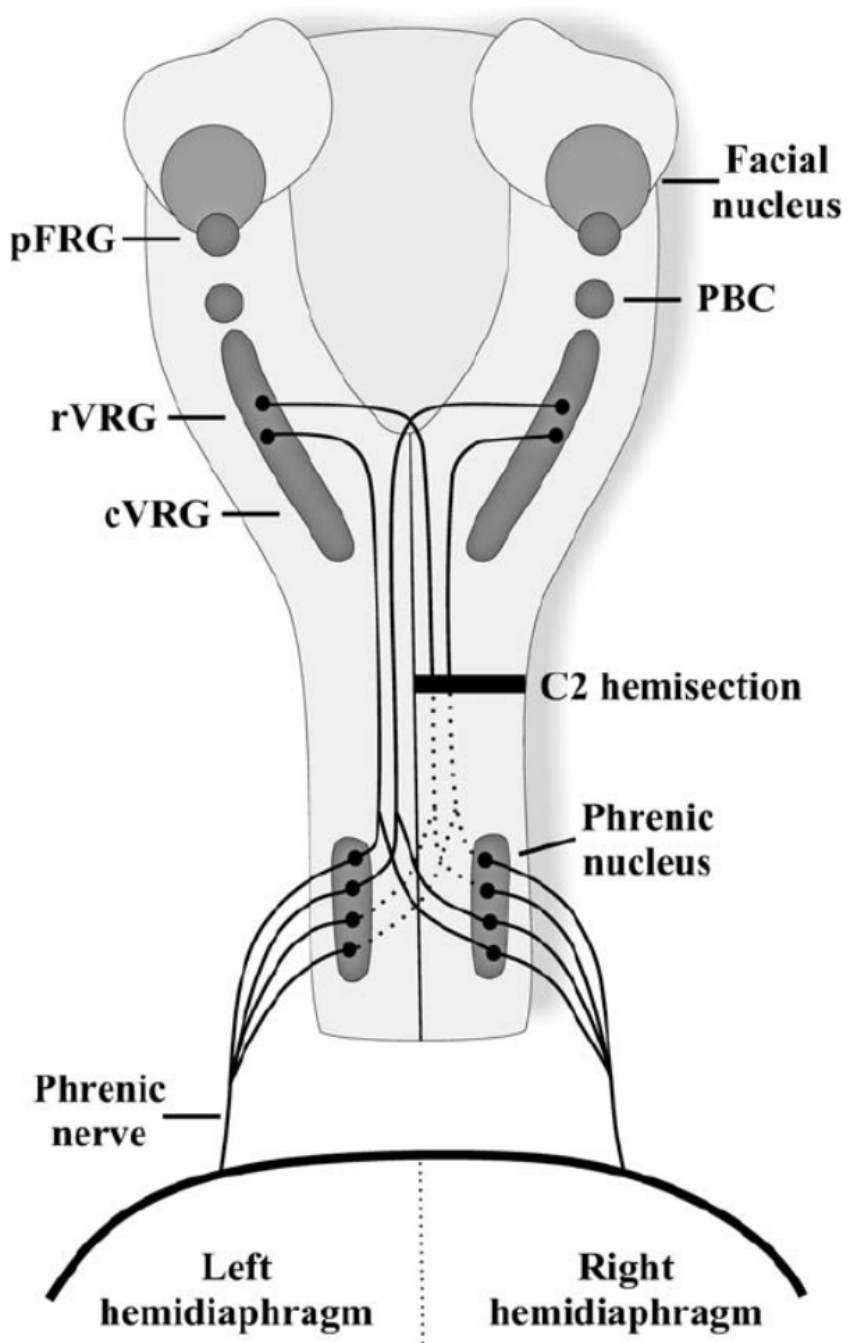

Legend: pFRG: parafacial respiratory group; rVRG: rostral ventral respiratory group; cVRG: caudal ventral respiratory group; PBC: preBotzinger complex.

Reproduced with permission. Effect of Spinal Cord Injury on the Respiratory System: Basic Research and Current Clinical Treatment Options. Beth Zimmer et al. J Spinal Cord Med. 2007; 30:319-330

Figure 1. Schematic drawing of the major respiratory neural centers and pathways in the rat. Respiratory rhythm generation is formed by neurons located within the $\mathrm{pFRG}$ and the PBC. Impulses are transmitted to premotor neurons located within the VRG with inspira-tory neurons located predominantly in rVRG and expira-tory neurons localized to cVRG. Premotor neurons project either unilaterally or bilaterally down to phrenic motor neurons located on both sides of the spinal cord (C3-C6). Crossed respiratory axons (arising from VRG neurons bilaterally) have been localized at the level of the phrenic nucleus and contribute to the expression of the crossed phrenic phenomenon. Phrenic axons from each side of the spinal cord form the phrenic nerve, which projects to each half of the diaphragm. 


\section{A Normal}
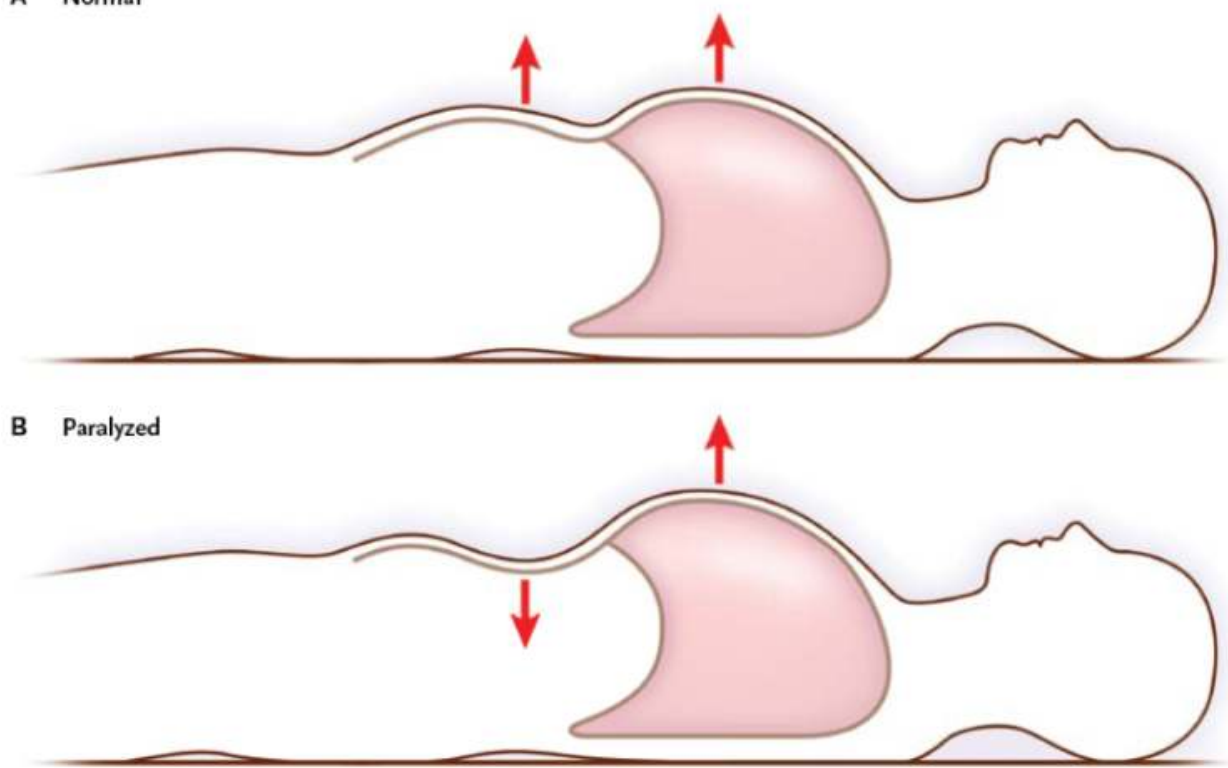

Reproduced with permission. Dysfunction of the Diaphragm. McCool, MD et al. N Engl J Med 2012; 366:932-42

Figure 2. Comparison of Rib-Cage and Abdominal Motion in Normal and Paralyzed Diaphragms. Panel A shows how normal diaphragmatic contraction results in an outward motion of the abdomen and rib cage (arrows). Panel B shows how diaphragmatic paralysis results in a paradoxical inward motion of the abdomen (down-ward-pointing arrow) during inspiration. The accessory inspiratory muscles contract, lifting the rib cage (upward-pointing arrow) and lowering the intrathoracic pressure. This change causes the flaccid diaphragm to move in a cephalad direction and the anterior abdominal wall to move inward.

cause virtually complete paralysis of both the inspiratory and expiratory muscles with mechanical ventilator dependency $(5,6)$. It may be possible to maintain neuromuscular transmission and functional properties of diaphragm muscle fibers after spinal cord injury when motoneurons are not directly injured (1). More than $80 \%$ of injuries are traumatic in nature, and up to $1.5 \%$ of such injuries are sustained in children. Many of these patients cannot be eventually weaned off mechanical ventilation, and require chronic ventilator support in as much as $4 \%$ of the cases $(4,6,7,8)$. In the United States more than 100,000 tracheostomies are performed for patients who are unable to be weaned from ventilator $(8,9)$. In most of these cases the lungs, chest wall, and respiratory muscles are usually physiologically normal (4). Acute SCI is complicated by respiratory complications in $67 \%$ of cases with pneumonia being the most common cause of death $(6,9,10)$. The C1-C4 injury group had pneumonia in $63 \%$ of cases at a mean interval of 30 days post-injury. Patients with high level lesions associated with bilateral diaphragm paralysis cannot achieve and sustain a vital capacity of $5 \mathrm{~mL} / \mathrm{Kg}$ (10). Controlled mechanical ventilation leads to rapid atrophy of the diaphragm. Within 18 hours of mechanical ventilation there is a decrease in type I muscle fibers with conversion to less functional fast-twitch type IIb muscle fibers (8). 


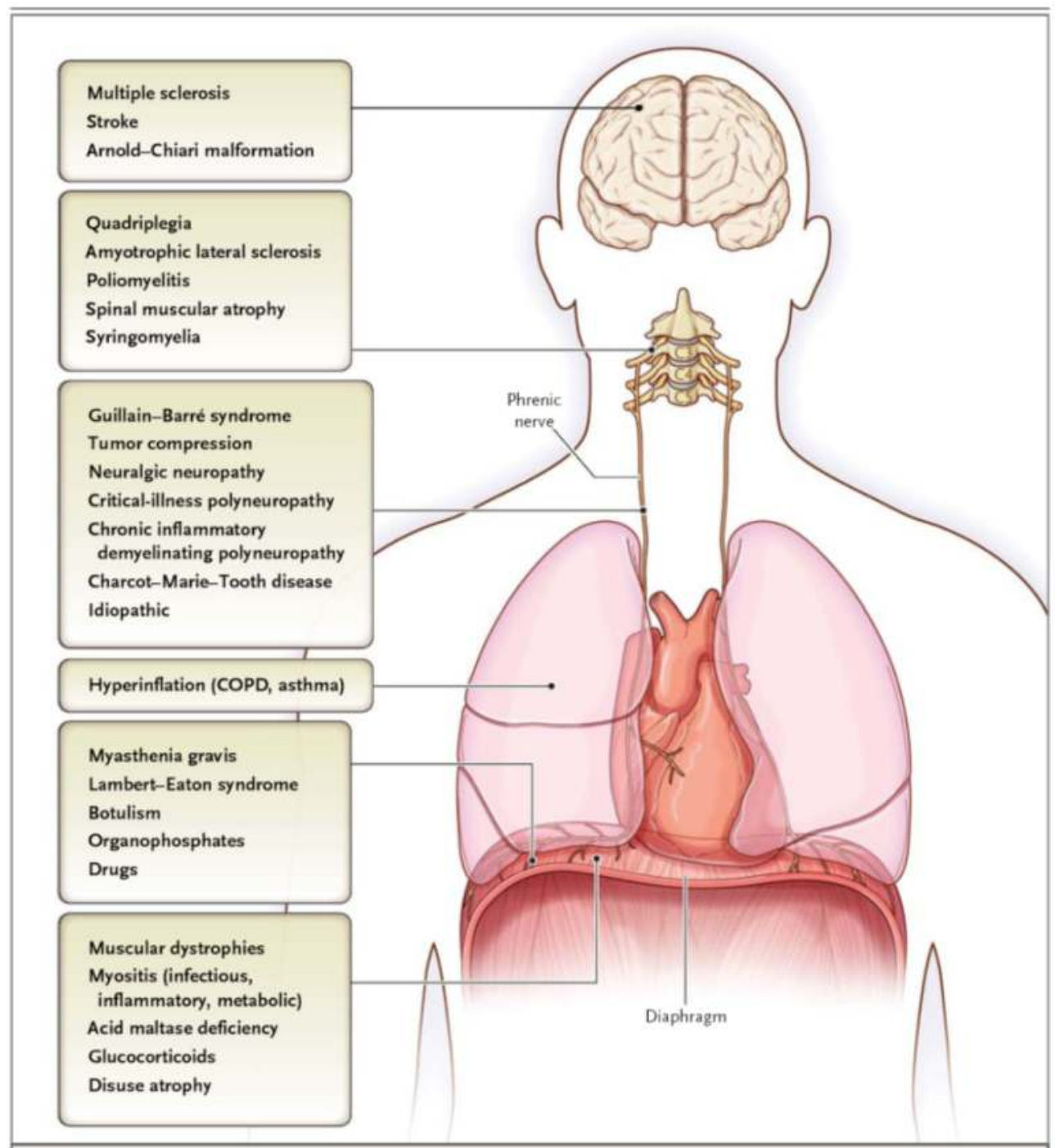

Reproduced with permission. Dysfunction of the Diaphragm. McCool, MD et al. N Engl J Med 2012; 366:932-42

Figure 3. Causes of Diaphragmatic Dysfunction, According to Level of Impairment. Disorders that occur at various regions in the body can lead to diaphragmatic dysfunction. COPD denotes chronic obstructive pulmonary disease.

Beyond one year the chances of recovery of fully independent breathing is probably less than $5 \%$ of patients with complete high tetraplegia (10). Loss of diaphragm function reduces survival. It is estimated that a 15 year-old who sustains a high SCI and survives the first 24 hours, the need of mechanical ventilation decreases life expectancy by 21 years (7); in the case of a 40 year-old patient with tetraplegia and ventilator dependence who survives the 
first 24 hours life expectancy is 7 years. It is dramatically reduced to 1.8 years in a 60 yearold with SCI $(8,9)$.

Mechanical ventilation in tracheostomy patients has several complications such as speech difficulties, reduced mobility, difficulty with transfers, social limitations, loss of sense of smell, equipment malfunction, increased respiratory infections, and increased cost of care approaching $\$ 200,000$ a year $(7,8,10,11)$.

Central Alveolar Hypoventilation Syndrome (CAHS) or "Ondine's curse" is extremely rare condition that shares the ventilatory failure pattern along with preserved lung functions, normal chest wall mechanics, and physiologically normal respiratory muscles. It is characterized by failure of the autonomic control of breathing during sleep (12). The term originates from a German myth about Ondine, an oceanic nymph who punished her unfaithful lover with the loss of all movements that did not involve his conscious will. Thus, he was condemned to remain awake forever to be able to breathe $(12,13)$. Blunted response of the respiratory center to carbon dioxide leads to hypoventilation and central apneas resulting in hypercarbia and hypoxemia; typically exacerbated during periods of sleep. Pulmonary hypertension and cor pulmonale lead to death (12).

Besides trauma, other causes of phrenic nerve involvement leading to chronic diaphragm dysfunction include vasculitis, neuralgic amyotrophy (14).

Amyotrophic lateral sclerosis is a relentlessly progressive neuromuscular disease of unknown origin that affects both upper and lower motor neurons causing weakness, hyperreflexia and atrophy (15). The incidence is 0.2 to 2.4 cases per 100,000 populations; it is most commonly diagnosed in middle age, affects more men than women. Life expectancy of ALS is 3 to 5 years after diagnosis (15). The major cause of mortality in patients with ALS is respiratory failure because of the progressive decline of 3-5\% per month of Forced Vital Capacity (FVC) due to progressive loss of motor neurons leading to respiratory muscle weakness. Phrenic nerve pacing is a novel method being investigated to treat the diaphragm involvement in ALS (11).

Diaphragm pacing is a technique of artificial ventilation using electrical stimulation of the phrenic nerves to produce diaphragm contraction, providing many ventilator-dependent tetraplegic patients with freedom from mechanical ventilation $(16,17,18,19)$. Compared with mechanical ventilation, phrenic pacing provides more natural breathing dynamics, reduces the occurrence of respiratory infection, and improves quality of life. Additionally earlier home discharge and lower infections drive down health care costs (20). Diaphragm muscle pacing, phrenic nerve pacing, and combined intercostal and unilateral diaphragm pacing techniques are currently been used to wean patients from ventilators and reduce the incidence of infection, atelectasis and respiratory failure (6).

Diaphragm pacing is conducted with low frequency electrical stimulation at a slow repetition rate to condition the diaphragm muscle against fatigue (21). 


\section{History}

The use of electricity for therapeutic purposes dates back to $15 \mathrm{AD}$, when Scribonious Largus, a court physician to the Roman emperor Claudius began using electric shocks from the torpedo ray fish to treat gout pain and headaches (22).

Sophisticated techniques for electrical stimulation of excitable tissue in neuromuscular disorders have been developed over the past three decades. Caldani in 1786 was probably the first to note movement of the diaphragm upon electrical stimulation of the phrenic nerve. In 1818 Ure applied electricity to the phrenic nerve of a recently hung criminal producing "strong and laborious respirations". In 1873, Hufeland proposed stimulating the phrenic nerve to treat asphyxia in neonates. During $19^{\text {th }}$ century attempts were made to apply direct electrical stimulation to the diaphragm or to phrenic nerves in the neck $(21,23,24)$.

By 1878, phrenic nerve stimulation was accepted as a rational procedure. Ziemssen used placement of large well-moistened sponges firmly over phrenic nerves at the outer borders of sternocleidomastoid muscles at the lower end of the scalene muscles to electrically stimulate the nerves (21).

The advent of mechanical ventilation led to abandonment of efforts at diaphragm stimulation as a means of supporting ventilation except for a few case reports. Sarnoff in 1948 that provided adequate ventilation with transcutaneous phrenic nerve stimulation for up to 52 hours in paralyzed patient with cerebral aneurysm. He also demonstrated this in bulbar poliomyelitis $(3,4,16,21,25)$. Sarnoff et al experimented extensively with electrical stimulation of the phrenic nerve and demonstrated that submaximal electrical stimulation of only one phrenic nerve could affect normal oxygen and carbon dioxide exchange; they called this method electrophrenic respiration (EPR) (26). In 1966 Glenn and coworkers first treated a patient with chronic ventilatory failure, using a radiofrequency system with an implantable receiver and electrode but an external power source. This technique was adopted as the gold standard of clinical application for more than 20 years. The surgery involved placement of electrodes on the phrenic nerve in the neck and thorax through a thoracotomy $(9,16,22,25,27,28)$. The initial device consisted of an external battery-powered transmitter with an antenna placed on the skin and a radio receiver placed subcutaneously beneath the antenna (3). See Figure 4.

Successful use of implantable pacemakers for the heart has prompted further interest in development of electrical stimulation of excitable tissues including the diaphragm. Improvements in pacemaker equipment design and intensive-care technology have increased the chances of using diaphragm pacing for the treatment of respiratory failure due to diaphragm paralysis (29). By 2004 approximately 1600 patients worldwide had been implanted with one of these pacing systems during last 35 years $(9,22)$.

In multicenter trials of SCI patients, $98 \%$ of patients were liberated from their ventilators with the diaphragm pacing system, which led to US Food and Drug Administration (FDA) approval for this indication in 2008. 


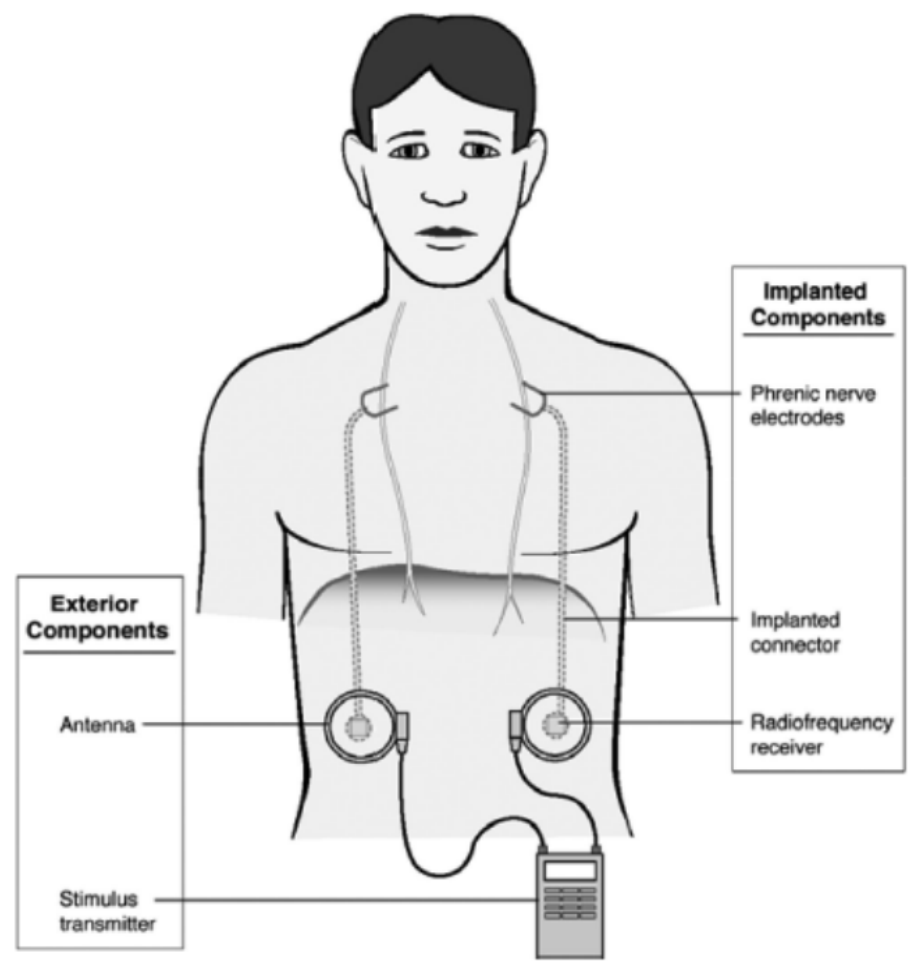

Reproduced with permission by Functional Electrical Stimulation Therapies after Spinal Cord Injury. David Gater et al. NeuroRehabilitation 28 (2011)231-248

Figure 4. Functional electrical stimulation of the diaphragm for respiration

\section{Indications}

The accepted indications for phrenic nerve pacing are respiratory paralysis after Cervical $\mathrm{SCI}$ above the origin of the phrenic nerve neurons (C1-C2) that are ventilator-dependent and the chronic Central Hypoventilation Syndrome associated with central apneas $(2,13,23,26,30,31)$. Diaphragm pacing has also been tried in chronic obstructive pulmonary disease (COPD), ALS $(15,32,33)$ and chronic hiccup $(34,35)$. Glenn et al elaborated on indications for the use of these pacemakers.

A diaphragmatic pacemaker may be considered for:

1. Chronic, respiratory failure requiring invasive mechanical ventilation via permanent tracheostomy on either a temporary, intermittent or continuous basis.

2. A central neurological cause for the respiratory failure such as

a. Alveolar hypoventilation, either primary or secondary to a brainstem disorder.

b. Interruption of neuronal conduction at the upper cervical level, above the C3 level

3. Integrity of the intrathoracic section of the phrenic nerve 
4. Acceptable pulmonary function

5. Normal level of consciousness (36).

Acquired CAHS has been described with medullary tumors, infection (particularly poliomyelitis), upper cervical trauma, some mitochondrial diseases, degenerative diseases (eg multiple sclerosis), and non-specific ischemic insults (12).

In the case of ALS patients, the goal is to initiate phrenic pacing before tracheostomy is required in order to maintain diaphragm strength. This will help convert diaphragm muscle fibers from the fast-twitch type IIb to slow-twitch type I and perhaps have a trophic effect that allows the phrenic motor neurons to live longer (32).

Diaphragm pacing has also been tried for Arnold Chiari malformation, meningomyelocele, neurofibromatosis, incomplete $\mathrm{C} 3-\mathrm{C} 4$ fractures, $\mathrm{C} 4-\mathrm{C} 5$ fractures with ascending paralysis to C2-C3 level, radiation induced phrenic nerve injury (3).

\section{Evaluation}

Diaphragm pacing is a costly undertaking, and depending on the specific technique of implantation, variable amounts of risks are associated with the surgical procedure. Therefore, potential candidates should be carefully screened and meet specific eligibility criteria. Initial evaluation should thoroughly assess psychological factors $(4,37,38)$. Some centers will even wait a year after injury to consider diaphragmatic pacemaker implantation (9). Patients with lesions of lower motor neurons, of the phrenic nerve itself or its roots, or with diaphragm weakness not due to disuse atrophy should not be consider for pacing $(28,39)$.

The ideal candidates for phrenic nerve pacing are those with normal phrenic nerves, diaphragms, and lungs $(5,21)$. Pacing may not be effective in patients with lesions involving the segment of the cord C 3 and C5 as some or all of the phrenic nerve cell bodies or lower motor neurons may be destroyed (21). Glenn et al reported that of 77 cases selected, 48 patients had defects in respiratory control, and 29 had lesions of the upper motor neurons of the phrenic nerve (21). For patients who do not have intact phrenic nerves, conventional or intramuscular diaphragmatic pacing is not an option (5).

Once suspected, diaphragmatic dysfunction can be confirmed by a number of tests. Chest radiographs may reveal elevated diaphragms and basal subsegmental atelectasis. Sniff tests are not useful to diagnose bilateral diaphragmatic paralysis $(2,38)$. Pulmonary function tests, especially measurements of upright and supine vital capacity, are non invasive and may support or refute the diagnosis(2,14,38). Arterial blood gas values during mechanical ventilation $(23,36,38)$ and a sleep study when possible, add diagnostic information (23).

Maximal static inspiratory pressure and sniff nasal inspiratory pressure have limitations due to patient effort. Both tests are markedly reduced to less than $30 \%$ of the predicted value, in those with bilateral diaphragm paralysis, as well as tetraplegia patients $(2,6)$. 
Other tests to assess diaphragmatic function could be invasive like transdiaphragmatic pressure (Pdi) or noninvasive like ultrasonography (2). Measurement of Pdi provides the difference between gastric and esophageal pressures during tidal breathing, maximal sniff maneuvers (sniff Pdi), maximal inspiratory efforts against a closed glottis (Pdi max), or transcutaneous electrical or magnetic stimulation of the phrenic nerve (twitch Pdi) $(2,25,38)$.

A twitch Pdi greater than $10 \mathrm{cmH}_{2} \mathrm{O}$ with unilateral phrenic-nerve stimulation or greater than $20 \mathrm{cmH}_{2} \mathrm{O}$ with bilateral phrenic-nerve stimulation also rules out clinically significant weakness. Ultrasonography of the diaphragm at its zone of apposition with the rib cage shows thickening of the diaphragm reflecting shortening during inspiration. Loss of thickening is diagnostic of diaphragmatic paralysis (2). Ultrasonography is ideal in children because it is noninvasive, painless, safe and portable. Image quality is superior in children compared with adults because of the small body size and lower body fat (40).

Electromyography (EMG) of the diaphragm during tidal breathing or after phrenic nerve stimulation is limited by technical issues of electrode placement, "crosstalk" from adjacent muscle groups, and variable distance of electrodes to muscle related to subcutaneous thickness when electrodes are placed at standardized places like seventh and ninth intercostal spaces $(4,18)$.

Phrenic nerve integrity must be demonstrated by EMG twitch of diaphragm contraction in response to electrical stimulation prior to considering diaphragmatic pacemaker insertion. This is tested by cervical magnetic stimulation and/or electrical stimulation of the phrenic nerve in the neck $(20,21,36,38,39)$. However a recent French series which looked at long term outcomes, found benefit in phrenic nerve pacing even in patients who had injuries dating back several years. $(20,39)$. EMG response of the diaphragm is considered normal if the latency was within normal range between 5.5 and $6.5 \mathrm{~ms}$ for cervical magnetic stimulation, and between 6.5 and $8 \mathrm{~ms}$ for electrical stimulation. In patients on prolonged mechanical ventilation, due to diaphragm atrophy, conduction times may be prolonged $(4,5,20)$. Fluoroscopic evaluation of diaphragm movement during phrenic nerve stimulation, is useful to exclude both false-positive and false-negative results of phrenic nerve stimulation. The diaphragm should descend at least 3 to $4 \mathrm{~cm}$ during stimulation as visualized fluoroscopically $(4,23)$. Phrenic nerve function can also be assessed by measurements of transdiaphragmatic pressure Pdi $(4,39)$. See Figure 5.

\section{Surgical implantation techniques}

The goal of any diaphragmatic pacing system is to provide safe and effective activation of the diaphragm, sufficient to provide adequate ventilation to meet metabolic requirements (4).

In the past the pacemaker electrodes were placed at the cervical area (25); but is presently discouraged for several reasons. Cervical phrenic nerve stimulation may result in incomplete diaphragm activation in the presence of an accessory branch from a lower segment of the cervical spinal cord that joins the main trunk of the phrenic nerve in the lower neck region or thorax.(4). 


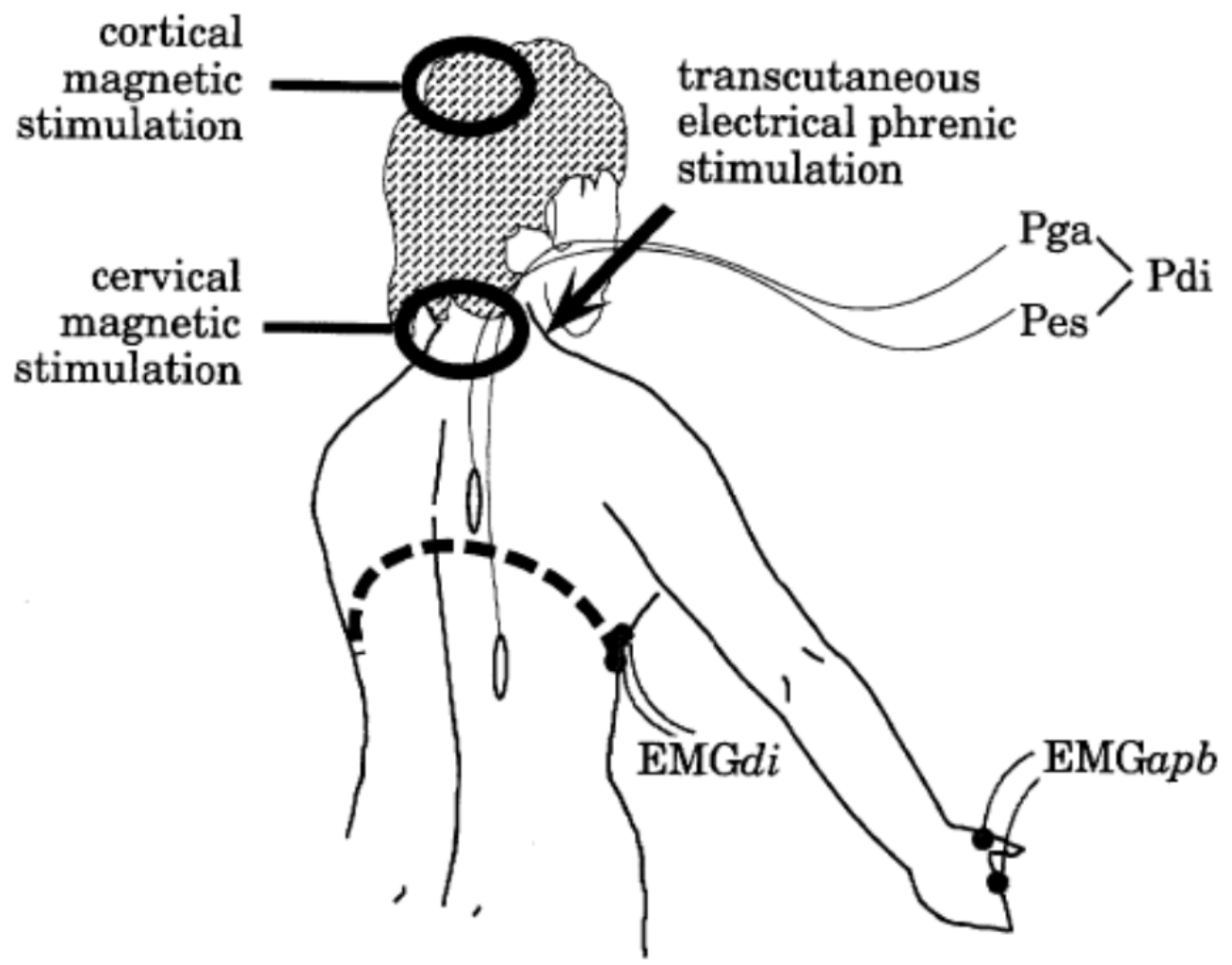

Reproduced with permission. Assessment of the Motor Pathway to the Diaphragm using Cortical and Cervical Magnetic Stimulation in the Decision-making process of phrenic Pacing. Thomas Similowski et al. Chest 1996; 110:1551-57

Figure 5. Schematic representation of the experimental setup used to study the patients. Diaphragmatic EMG (EMGdi), esophageal pressure (Pes), and gastric pressure (Pga) were measured in response to cortical and cervical magnetic stimulation, and to transcutaneous electrical stimulation of the phrenic nerve in the neck. Pdi was derived from Pes and Pga. An EMG ofthe APB (EMGapb) was used as control in some patients.

In Glenn's series, bilaterally thoracotomy procedures were performed 10 to 14 days apart. Once phrenic nerves were exposed surgically the platinum electrodes were placed directly in contact with the perineurium of the nerve in a tunnel behind the nerve that was large enough to allow free movement of the electrode within it. Then, the electrodes were connected to the receiver in the subcutaneous pocket were it was tested prior to skin closure (21).

Le Pimpec-Barthes et al successfully used Video-assisted thoracic surgery (VATS) for the implantation. The endoscopic camera was inserted in the pleural space through a second intercostal space incision. Careful dissection of the phrenic nerve was carried out in front of the vena cava on the right and in the pulmonary hilum on the left. Then quadripolar electrodes were implanted in both sides, 2 above and 2 below the nerve. Each Teflon patch 
was sutured to pleura or pericardium to prevent dislodgement. Then wires were brought out through a mini-thoracotomy, and tunneled under the pectoralis major muscle to a subcutaneous pocket. The wires were then connected to the subcutaneous radiofrequency receivers on each side. Intraoperative testing confirmed correct placement. No nerve injury or other significant intraoperative complications were observed (20). Robotic surgical placement has also been tested successfully including in patients with tetraplegia (4).

Another minimally invasive approach advocated intramuscular placement of the stimulating electrodes in the diaphragm laparoscopically $(5,9)$. In this method, identification of motor points of the phrenic nerves was done by a mapping procedure which involved electrical stimulation each hemidiaphragm with a probe at several locations. Two electrodes were placed in each diaphragm to stimulate all branches of the phrenic nerve maximally at the point where stimulation evoked the best motor response of the diaphragm $(5,9,11)$. After testing all 4 wires are brought out through the epigastric port, and then tunneled to the chest where they were connected to the transcutaneous exiting wires. Onders emphasized that the accurate identification of motor points is crucial for successfully pacing the diaphragm (9). DiMarco et al described further improvement of the technique with coarse mapping and direct visualization of diaphragmatic contraction $(4,15)$. A significant difference was noted in patients with ALS whose diaphragms had weaker contractions with the typical mapping stimulation.-Nevertheless, the laparoscopic procedure can be safely performed at multiple independent hospital sites for ventilator-dependent tetraplegia and ALS (11). Laparoscopic approach has cost and simplicity advantages and can be performed on an outpatient basis. In addition there is no manipulation of the phrenic nerve by itself (17).

Assouad et al have tried placing the electrodes through a cervical incision thoracic endoscopic surgery (CITES). Here a mediastinoscopy is followed by bilateral pleurotomies, and then a gastroscope is used in the pleural space to locate the phrenic nerve penetration points at the dome of the diaphragm. Finally needle and electrode hooks were placed close to the entry point of the phrenic nerves (19).

There are three commercially available diaphragmatic pacing systems depicted in table 1: a) Avery Biomedical Devices, b) Atrotech OY, and c) Medimplant systems. Each system is designed for lifetime use (4)

\section{Settings}

After surgical implantation, a period of 2 weeks should elapse before initiation of diaphragm pacing $(20,28,35)$. The diaphragm is weakened from prolonged mechanical ventilation, therefore, at the beginning a process of conditioning is carried through gradual lengthening of periods of stimulation $(28,38)$. Thus, a strategy of minimizing diaphragm fatigue during pacing should involve the gradual conditioning with low-frequency stimulation and a slow respiratory rate (29). Diaphragmatic conditioning is a process by which the fast-contracting fibers (anaerobic, highly glycolytic fibers), which are highly susceptible to get fatigued, are converted to slow-contracting fatigue-resistant (aerobic oxidative fibers), which enable permanent ventilatory support using diaphragmatic pacing $(4,36)$. 


\begin{tabular}{lllll}
\hline Device & $\begin{array}{l}\text { AVERY I-107A } \\
\text { (superceded) }\end{array}$ & AVERY I-110A & ATROTECH & MEDIMPLANT \\
\hline Receiver size (mm) & 46 (diam) $\times 16$ & 30 (diam) $\times 8$ & 49 (diam) $\times 8.5$ & $56 \times 53 \times 14$ \\
Battery life (hr) & 160 & 100 & $\begin{array}{l}160-320(12 \mathrm{~V}) \\
8(9 \mathrm{~V})\end{array}$ & 24 \\
Controller + battery weight $(\mathrm{kg})$ & 3.6 & 0.54 & $0.45+0.6(12 \mathrm{~V})$ & $0.8+0.62$ \\
Controller size (mm) & $179 \times 114 \times 97$ & $146 \times 140 \times 25$ & $185 \times 88 \times 28$ & $170 \times 130 \times 51$ \\
Breaths/min & $10-50$ & $6-24$ & $8-35$ & $5-60$ \\
Pulse interval (ms) & $35-170$ & $40-130$ & $10-100$ & $25-170$ \\
Pulse width (microsec) & 150 & 150 & 200 & $100-1000$ \\
Sigh possible & yes & yes & yes & yes \\
Electrodes & Monopolar; bipolar & Monopolar; bipolar & Quadripolar & Quadripolar \\
$\begin{array}{l}\text { No. of receivers to stimulate both } \\
\text { hemidiaphragms }\end{array}$ & 2 & 2 & 2 & 1 \\
\hline \hline
\end{tabular}

Reproduced with permission. Electrical Stimulation to Restore Respiration. Journal of Rehabilitation Research and Development. Vol 33. N 2. April 1996: 123-132

Table 1. Technical features of phrenic nerve stimulators

While on mechanical ventilation, many patients are generally maintained with large tidal volumes. Diaphragmatic pacing is likely to generate smaller tidal volumes leading to respiratory acidosis therefore ventilator adjustments are needed initially (4).

Glenn initiated 5 minutes per hour of stimulation with low frequency stimulation $(11 \mathrm{~Hz})$ at slow rate (8 to 10 per minute). Tidal volumes at the beginning and end of each stimulation period were measured. Once tidal volumes at the end of stimulation period were at least $75 \%$ of the beginning volumes, the length of pacing was progressively increased by one to two minutes per hour until the diaphragm was paced 30 minutes per hour. After that, periods of pacing were increased by 5 minute per hour daily. Mechanical ventilation was discontinued during pacing once minute volume was adequate $(>5 \mathrm{~L} / \mathrm{min}$ in adults). Diaphragm conditioning is performed during daytime. Each hour of pacing with good minute volume is followed by an hour of rest on the ventilator. Pacing duration is incrementally increased by 15 minutes per session until 6 hours are reached. These periods are progressively extended to 8, 10 and 12 hours. When pacing on each side has been extended to 12 hours, while producing satisfactory minute volumes and normal arterial blood gases, the patient can be completely removed from mechanical ventilation. This weaning process usually takes 2 to 3 months, which is the time frame required for diaphragmatic fiber recovery $(21,28,36,38,41)$. A considerably longer period of conditioning is required in children because of immaturity of respiratory components (21). Every few weeks the pulse frequency and respiratory rate are decreased until the lowest values were reached at which ventilation was still adequate (41). Once the maximal stimulation time has been established, several schedules for maintenance can be followed. For patients who respond normally to stimulation of either side of the diaphragm, they recommended pacing each side alternately, 12 hours at the time (28). The voltage required to effect minimal (threshold) and maximal diaphragm motion during pacing was confirmed or adjusted about once a month (41). Alternatively uninterrupted simultaneous pacing of both hemidiaphragms, using a low-frequency stimulation $(7-8 \mathrm{~Hz})$ 
with a respiratory rate of 5 to 9 per minute may be appropriate for adult patients (41). In general the transition from mechanical ventilation to chronic full-time pacing must be individualized for each patient (4). Occasionally this weaning process can be performed with a home-based ventilator weaning and conditioning program $(22,42)$.

Until full-time pacing is achieved, a tracheostomy needs to remain in place, even after discontinuation of positive pressure ventilation to facilitate intermittent suctioning. This also serves as a back up to resume mechanical ventilation in case of pacemaker failure or other issues like the lack of coordination between the paced diaphragm, the upper airway muscles, and the accessory respiratory muscles in tetraplegics $(28,34)$.

In patients with CAHS, upper airway resistance is augmented by diaphragmatic contraction or diminished upper airway muscle activity and may be induced by diaphragm pacing during sleep $(34,43)$. In this population without muscle paralysis, pacing can be more aggressively initiated, 8 to 10 hours nightly by the third week postoperatively. Moreover, CAHS patients should have baseline sleep study with and without pacing to assure adequacy of pacing and identify any upper airway obstruction (21).

ALS patients begin conditioning programs by five 30-min pacing sessions a day. If ALS patients develop hypoventilation, the amount of time paced can be increased and then used at night. Continuous positive airway pressure (CPAP) or noninvasive positive-pressure ventilation may still be needed to maintain a patent upper airway (11).

Pacemaker function should be monitored on a routine basis and emergently in situations in which patients complain of difficulty breathing. Tidal volumes can be easily measured by spirometry; both electrodes stimulating each diaphragm can be independently evaluated. Pulse oxymetry as well end-tidal $\mathrm{PCO}_{2}$ should also help to assess ventilation (4).

\section{Benefits}

Ventilation by phrenic nerve pacing appears to provide both health and lifestyle advantages to the user. Freedom from positive-pressure ventilator reduces the risks of ventilatorassociated problems like tracheomalacia, infections, bleeding, barotraumas, and tracheoesophageal fistulas $(4,22,23)$.

Other ventilator-related disasters are also prevented like sudden death from accidental disconnection from ventilator, or mechanical ventilation device failure (23).

Olfaction was altered because nasal fossae were bypassed during positive-pressure ventilation on tracheostomy patients. Thus, improved speech and sense of smell recovery contribute to a better perception of quality of life in patients with successful diaphragmatic pacing $(8,23,44)$.

Phrenic nerve pacing allows negative-pressure ventilation, leading to a decrease in respiratory infections, in addition to improved patient comfort (8).

Liberation from ventilation also leads to reduced weight of their wheelchair and simplified transfers as well as elimination of social embarrassment associated with ventilator dependency $(4,7)$. 
Additionally, patients with pacing systems felt an overall improvement in their well being by reducing fatigue, depressed mood state and sleep disruptions that result from prolonged mechanical ventilation(7). A case report has demonstrated clinical evidence of improvement in right heart failure from pacing, and a fall in red cell volume and pulmonary artery pressures (33). Better ventilation of the posterior lobes minimizes atelectasis and improves cardiac function by increasing venous return (7).

Interestingly, in patients with ALS, diaphragmatic pacing significantly reduced the rate of decline of FVC from $2.4 \%$ preimplant to $0.9 \%$ per month postimplantion extrapolating to a 24-month improvement in survival (32).

\section{Complications}

A major complication is the iatrogenic injury to the phrenic nerve during operative implantation of phrenic nerve electrode and subsequent pacemaker failure $(4,30)$. In patients with thoracoscopic pacemaker implantation the most feared complication is phrenic nerve entrapment (36).

In addition to complications common to all surgical procedures, laparoscopy may be associated to pneumothorax, capnothorax $\left(\mathrm{CO}_{2}\right.$ tracking from the pressurized abdomen to the pleural space) and subcutaneous emphysema. Right shoulder pain may result from stimulation of phrenic nerve afferents $(11,27,28,45)$. All surgical procedures involving implantation of a foreign body carry some risk of infection, with rates of approximately $3 \%$ in case of phrenic nerve pacing (4).

Phrenic pacing can worsen or induce upper airway obstruction with central alveolar hypoventilation; therefore a patent tracheostomy is necessary during sleep $(4,29)$. Similarly in ALS patients that have severe bulbar weakness the upper airway can collapse during inspiration, thus needing a tracheostomy or CPAP treatment (15).

DiMarco reported one subject developed intermittent aspiration of food during meals, due to large negative airway pressure generated during diaphragmatic contraction (27).

Potential long-term complications related to pacing include electrode dislodgement and breakage that will result in device malfunction $(22,27)$.

Pacemaker failure due to technical problems is always a possibility. Strong magnetic fields, such as magnetic resonance imaging can override the electronic system of diaphragmatic pacemakers (4).

\section{Outcomes}

Diaphragmatic pacing can allow patients the benefits of negative pressure breathing. They have been used safely up to 19 years post injury in individuals with tetraplegia (7). All of these patients were able to tolerate outpatient surgery without perioperative morbidity.

Retrospective assessments have indicated a correlation between excess mortality and neurological level of injury, older age at injury, and injury at earlier calendar years (5). 
The problems associated with bilateral phrenic nerve pacing in infants and children are related more to cor pulmonale, as result of hypoventilation, than the mechanics of pacing itself, which can lead to uncertain outcomes (13).

It has been shown to improve quality of life and to extend survival in patients with advanced respiratory muscle weakness (12).

Carefully selected patients should undergo phrenic nerve pacemaker placement by experienced surgeons. They need to have individualized monitored pacing regimens with close follow up for best outcomes $(25,37)$.

The cost savings could be significant. The average cost of ventilator-dependent patient is three times that without a ventilator. During 2004 available phrenic nerve pacing devices with the hospitalization cost more than $\$ 120,000$. One of the patients in that series who was nursing homebound was able to save $\$ 13,000$ a month to Medicaid, because he no longer needed to be in a ventilator unit (9). Another series demonstrated that the first year investment for diaphragmatic pacing is paid off after three years. When costs of a respiratory infection are incorporated in the cost analysis, the diaphragm pacing becomes more cost effective in 1 year. (18).

Overall, patients with SCI treated with phrenic nerve pacing instead of standard mechanical ventilation experienced less airway infections, had reduced costs for single use airway equipment, had improved the quality of speech, had improved quality of life, and probably derived a mortality benefit (18).

Although the number of patients who may benefit from this technology is small, the potential improvement in quality of life is substantial $(46,47)$.

Garrido-Garcia and colleague found the mortality to be $22.7 \%$ (36). The problems associated with bilateral phrenic nerve pacing in infants and children are related more to cor pulmonale, as result of hypoventilation, than the mechanics of pacing itself (13).

Diaphragmatic pacemakers are safe to use in patients with cardiac pacemakers.

Patient satisfaction in recipients of diaphragm pacing was very high particularly in tetraplegic patients on ventilators (20).

\section{Conclusion}

Over the last fifty years, significant progress has been made in diaphragmatic pacing. Improvement in surgical techniques have reduced procedural complication during implantation Research in this field has relatively lagged behind other pacemakers such as cardiac devices presumably due to less financial motivation. Ideally a diaphragmatic pacemaker should synchronize with electrical signals from the central nervous system, and adapt to changing metabolic demands. Further development of such devices promises hope for patients with chronic respiratory failure for improved quality of life and possible mortality benefit at a reasonable cost. 
In conclusion this technique is efficient in liberating selected ventilator-dependent patients with respiratory paralysis and treating alveolar hypoventilation syndromes. Future clinical trials will increase acceptance of this method as an alternative form of treatment for respiratory failure in selected patients.

\section{Author details}

Jorge F. Velazco ${ }^{1,2}$, Shekhar Ghamande ${ }^{1,2}$ and Salim Surani ${ }^{1, *}$

Texas AEM University, Texas, USA

Scott $\mathcal{E}$ White Hospital, Temple, Texas, USA

\section{References}

[1] Neuromuscular adaptations to respiratory muscle inactivity. Carlos B. Mantilla; et al. Respir Physiol Neurobiol. 2009 Nov 30; 169(2): 133-140.

[2] Dysfunction of the Diaphragm. F. Dennis McCool, M.D; et al. N Engl J Med 2012; 366:932-942.

[3] Diaphragmatic Pacemaker. Salim Surani, MD. Modern Pacemakers - Present and Future. In-Tech. 2011.453-470.

[4] Inspiratory Muscle Pacing in Spinal Cord Injury: Case Report and Clinical Commentary. Anthony F. DiMarco,MD; et al. J Spinal Cord Med 2006; 29:95-108.

[5] Respiratory Dysfunction and Management in Spinal Cord Injury. Robert Brown, MD; et al. Respir Care. 2006 Aug; 51(8): 853-870.

[6] Effect of Spinal Cord Injury on the Respiratory System: Basic Research and Current Clinical Treatment Options. M. Beth Zimmer, PhD; et al. J Spinal Cord Med. 2007; 30:319-330.

[7] Diaphragm Pacing Stimulation System for Tetraplegia in Individuals Injured During Childhood or Adolescence. Raymond P. Onders, MD; et al. J Spinal Cord Med. 2007; 30:S25-S29.

[8] Multicenter analysis of diaphragm pacing in tetraplegics with cardiac pacemakers: Positive implications for ventilator weaning in intensive care units. Raymond P. Onders, MD; et al. Surgery 2010; 148:893-898.

[9] Mapping the phrenic nerve motor point: The key to a successful laparoscopic diaphragm pacing system in the first human series. Raymond P. Onders, MD; at al. Surgery 2004; 136:819-826.

[10] Delayed diaphragm recovery in 12 patients after high cervical spinal cord injury. A retrospective review of the diaphragm status of 107 patients ventilated after acute spinal cord injury. T Oo; et al. Spinal Cord (1999) 37:117-122.

[11] Complete worldwide operative experience in laparoscopic diaphragm pacing: results and differences in spinal cord injured patients and amyotrophic lateral sclerosis patients. Raymond P. Onders; et al. Surg Endosc (2009) 23:1433-1440.

\footnotetext{
${ }^{*}$ Corresponding Author
} 
[12] Ondine's curse: anesthesia for laparoscopic implantation of a diaphragm pacing stimulation system. Ahtsham U. Niazi, MBBS; et al. Can J Anesth/J Can Anesth (2011); 58:1034-1038.

[13] Congenital central hypoventilation syndrome: A report of successful experience with bilateral diaphragmatic pacing. Michael Coleman; et al. Arch Dis Child. 1980 Nov; 55(11): 901-903.

[14] Diaphragm plication in adult patients with diaphragm paralysis leads to long-term improvement of pulmonary function and level of dyspnea. Michel I.M. Versteegh; et al. European Journal of Cardio-thoracic Surgery 32 (2007):449-456.

[15] Laparoscopic diaphragmatic pacer placement - a potential new treatment for ALS patients: a brief description of the device and anesthetic issues. Clifford A. Schmiesing MD; et al. Journal of Clinical Anesthesia (2010) 22, 549-552.

[16] Radio-Frequency Electrophrenic Respiration Long-Term Application to a Patient With Primary Hypoventilation. John P. Judson, MD; et al. JAMA Mar 18, 1968;203(12):129134.

[17] Phrenic Nerve Pacing in a Tetraplegic Patient via Intramuscular Diaphragm Electrodes. Anthony F. DiMarco; et al. Am J Respir Crit Care Med 2002; 166:1604-1606.

[18] Mechanical ventilation or phrenic nerve stimulation for treatment of spinal cord injuryinduced respiratory insufficiency. S Hirschfeld; et al. Spinal Cord (2008); 46:738-742.

[19] Minimally invasive trans-mediastinal endoscopic approach to insert phrenic stimulation electrodes in the human diaphragm: a preliminary description in cadavers. Jalal Assouad; et al. European Journal of Cardio-thoracic Surgery 40 (2011) e142-e145.

[20] Intrathoracic phrenic pacing: A 10-year experience in France. Francoise Le PimpecBarthes, MD; et al. J Thorac Cardiovasc Surg 2011;142:378-383.

[21] Diaphragm Pacing by Electrical Stimulation of the Phrenic Nerve. William W.L. Glenn, MD; et al. Neurosurgery 17:974-984, 1985.

[22] Functional electrical stimulation therapies after spinal cord injury. David R. Gater, Jr; et al. NeuroRehabilitation 28 (2011) 231-248.

[23] Electrical stimulation to restore respiration. Creasey, Graham, MD; et al. Journal of Rehabilitation Research and Development; Apr 1996; 33:123-132.

[24] Lung function in diaphragm pacing S.W. Epstein, MD; et al. CMA Journal Jun 9,1979; 120:1360-1367.

[25] Diaphragm pacing. John Moxham; et al. Editorial. Thorax 1988; 43:161-162.

[26] Central Hypoventilation; Long-term Ventilatory Assistance by Radiofrequency Electrophrenic Respiration. William W. L. Glenn, M.D et al. Annals of Surgery, Oct 1970; 172(4): 755-773.

[27] Phrenic Nerve Pacing Via Intramuscular Diaphragm Electrodes in Tetraplegic Subjects. Anthony F. DiMarco, MD; et al. Chest 2005; 127:671-678.

[28] Long-Term Ventilatory Support by Diaphragm Pacing in Quadriplegia. William W.L. Glenn, MD; et al. Ann Surg 1976; 183(5): 566-577.

[29] Problems Associated with Diaphragm Pacing. Takashi Nishino. Respiration 2002; 69:1213. 
[30] Twenty Years of Experience in Phrenic Nerve Stimulation to Pace the Diaphragm. William, W. Glenn, MD; et al. PACE (1980) Vol 9. pp 780-784.

[31] Eight-Year Follow-Up Study of a Patient with Central Alveolar Hypoventilation Treated with Diaphragm Pacing. Fumihiko Yasuma et al. Respiration 1998; 65:313-316.

[32] Amyotrophic lateral sclerosis: the Midwestern surgical experience with the diaphragm pacing stimulation system shows that general anesthesia can be safely performed. Raymond P. Onders, M.D; et al. The American Journal of Surgery (2009) 197, 386-390.

[33] Diaphragm pacing in ventilatory failure. S Lozewicz; et al. BMJ 1981, Oct 17; 283:10151016.

[34] The Swedish Experience in Phrenic Nerve Stimulation. Harald Fodstad. PACE, 10, January-February, Part II, 1987:246-251.

[35] Transesophageal Diaphragmatic Pacing for Treatment of Persistent Hiccups. David W. Andres, M.D; et al. Letter to Editor. Anesthesiology 2005; 102:483.

[36] Treatment of chronic ventilatory failure using a diaphragmatic pacemaker. H GarridoGarcia; et al. Spinal Cord (1998) 36:310-314.

[37] Long-Term Follow-Up of bilateral Pacing of the Diaphragm in Quadriplegia. John F. Elefteriades, MD; et al. Letter to the Editor. N Engl J Med 1992; 326:1433-1434.

[38] Transdiaphragmatic pressure in quadriplegic individuals ventilated by diaphragmatic pacemaker. Honesto Garrido-Garcia; et al. Thorax 1996; 51:420-423.

[39] Assessment of the Motor Pathway to the Diaphragm Using Cortical and Cervical Magnetic Stimulation in the Decision-making Process of Phrenic Pacing. Thomas Similowski, MD; et al. Chest 1996; 110:1551-1557.

[40] Ultrasound evaluation of piglet diaphragm function before and after fatigue. Keith C. Kocis; et al. J Appl Physiol 83:1654-1659, 1997.

[41] Ventilatory Support by Pacing of the Conditioned Diaphragm in Quadriplegia. William W.L. Glenn, MD; et al. N Eng J Med 1984; 310:1150-1155.

[42] Lo stimolatore frenico, valido supporto ventilatorio per l'assistenza domiciliare dei pazienti tetraplegici. Caso clinico. A. M. Giglio; et al. Minerva Anestesiol 2002; 68:567571.

[43] Conditioning of the diaphragm by phrenic nerve pacing in primary alveolar hypoventilation. Pearce G Wilcox; et al. Thorax 1988; 43:1017-1018.

[44] Diaphragm pacing restores olfaction in tetraplegia. D. Adler; et al. Eur Respir J 2009; 34: 365-370.

[45] Chest Pain and Diaphragmatic Pacing after Pacemaker Implantation. John L. Jefferies, MD; et al. Texas Heart Institute Journal. Volume 32, Number 1, 2005:106-107.

[46] Diapham pacing. Gerhard A Baer; et al. Correspondence. Thorax 1988; 43:743-744.

[47] Efficacy of Combined Inspiratory Intercostal and Expiratory Muscle Pacing to Maintain Artificial Ventilation. Anthony F. DiMarco; et al. Am J Respir Crit Care Med 1997; 156:122-126. 